\title{
مشكلات ترقية كفاءة الطلبة في اللغة العربية
}

\author{
Fadhilah Samhabib \\ IAIN Kendari \\ dilasamhab@gmail.com
}

\begin{abstract}
Competence is skills that are closely related to the effectiveness of person's performance in a particular field. Similarly in education, competence is the key to student success, and also the great competence of the majors chosen by the students will lead to increased quality of majors. Arabic Language Education Department in IAIN Kendari is a department that prepares students to become educators and scientists in Arabic language which has competence in Arabic were nice and had a high competitiveness. This research uses descriptive analysis method with qualitative approach based on case study design. The data collection is done by in-depth interviews, observation, documentation, and open questionnaire. The results showed (1) a strategy to increase student competence in Arabic language on Arabic language education department at IAIN Kendari still ineffective that was described by least of activities that support the increased student's competence in Arabic language and also its lack of consistency in implementing additional programs for students of Arabic language education department (2) the helping and resistoring factors to increase student's competence contained on lecturer's performance in the classroom, facilities of language learning, and student's motivation. lecturer's performance in the classroom has given many help to increase student's competence in Arabic, facilities to assist in learning the Arabic language still needs to be cared and activated again or making the new and interesting activities, and student's motivation in the study of Arabic is still lacking is due to the absence of another place for practicing the Arabic language competencies that they have acquired, and also the lack of student's interest in attention of learning at the classroom caused by the bored atmosphere of the class (3) the problem solving by formulating a strategy based on suggestions and feedback from lecturers and students in Arabic language education department is a path that can be taken by Arabic language education department in IAIN Kendari to improve student's competence in Arabic language.
\end{abstract}

Keywords : Problematics, Student's Competence, Arabic Language.

$$
\begin{aligned}
& \text { لدي الفرد1. موان الكفاءة هي مهم في تحديد } \\
& \text { جودة وبناح البشر في بحال الذي تعمّق فيها. } \\
& \text { سيتم التعرف على شخص ما في بجال إذا كان } \\
& \text { لديه الكفاءة الجيدة في هذا المحال. } \\
& \text { 1اوريل بحر الدين، مهارات التدريس نخو إعداد مدرس اللغة العربية } \\
& \text { الكفء، (مالانج : مطبعة جامعة مولانا مالك إبراهيم الإسلامية، } \\
& \text { 2011)، ص. } 64
\end{aligned}
$$

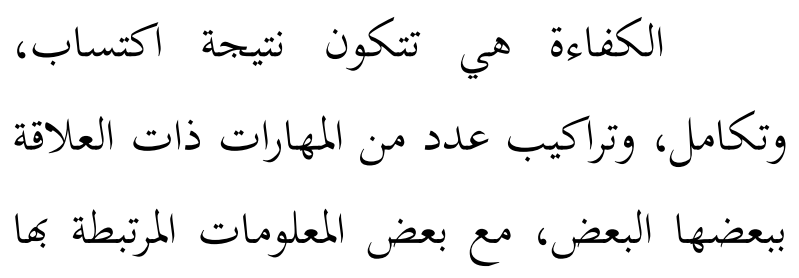


ولكن في الواقع لا يزال هناك نقص في

الانتباه إلى قسم تعليم اللغة العربية في الجامعة

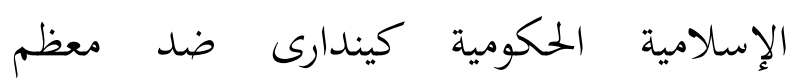
الاحتياجات الأساسية لطلبة قسم تعليم اللغة

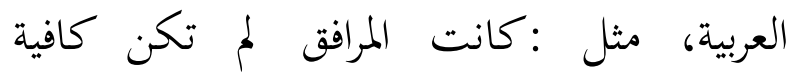
ومؤسسة اللغة التي لم تكن تحقيقها والاتساق في تشغيل برنامج أسبوعي للطلبة وقليل الأنشطة المتعلقة باللغة وخصوصا عن اللغة العربية مما تسبب على كفاءة طلبة قسم تعليم اللغة العربية لم يستطع ان يقالها جيدة.

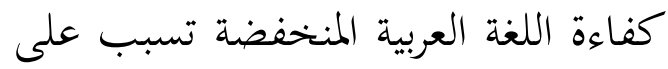
المتخرجين الذين لم تكونو قادرا على القول جودة

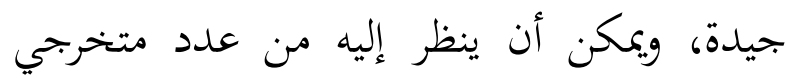
تعليم اللغة العربية في مدينة كيندارى الذين لم الم يستعب اللغة العربية كأساس العلوم التي وجبت لعبد على استيعاهما بطلبة قسم تعليم اللغة العربية.

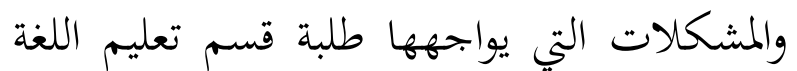
العربية هي:

1- لم يكونوا ماهرين في تكلم اللغة العربية 2- لم يكونوا متعمّق عن قواعد اللغة العربية ودقائق الشيئ عن اللغة العربية فيجعل الطلبة يصعب على فهم مقالات اللغة العربية

3- ان كفاءة متخرجي تعليم اللغة العربية

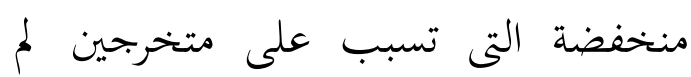
يستطعوا ان يتنافسوا بالمتخرجي الأخرين
في التربية ان الكفاءة هي شيئ الذي وجب على اهتمامها. وفي الجامعة، كفاءة الطلبة هي أحد المحددات من الإخراج الذي سيكون نقطة من النجاح وتحسين جودة جامعة. الجحامعة

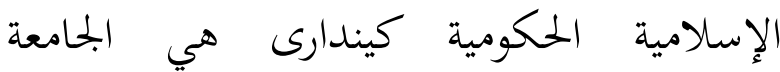
الإسلامية لما المسؤولية عن زيادة كفاءة الطلبة في بحالات مختلفة من الحقل الذي اتخذ من قبل

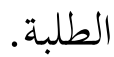
ولتسير التربية سيرا جميلا، فيكون القسم الموجود يستطيع ان ينتج الطلبة له كفاءة مناسبة

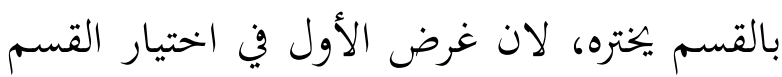
هو يركز تعلم الطلبة على ما اختراه. وقال بحمل

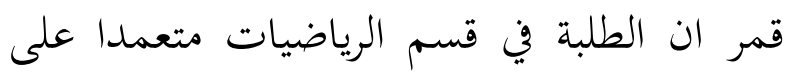
إستيعاب الرياضيات حين الانتهاء من دراسته ولو أقل يستعيب العلوم الدينية2. وبالمثل، فإن الطلبة الطناء من دراسته في قسم تعليم اللغة العربية، يجب أن تكون في

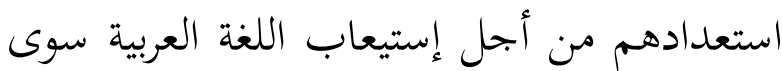
من العلوم التربوية .وهذا يسبب لاستيعاب اللغة الغة العربية هو واجب على الطلبة والمتخرجين من قسم تعليم اللغة العربية، لان في تصميم معلم اللغة العربية يحتاج كذلك على استخدام اللغة العربية سوى من استيعاب العلوم التربوية ويستطيع

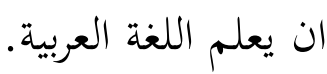

\footnotetext{
${ }^{2}$ Mujamil Qamar, Dimensi-dimensi Manajemen Pendidikan Islam, (Jakarta:PT Erlangga, 2015), hlm. 429
} 


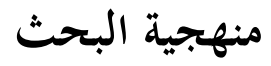

في هذا البجث يستخدم مدخل النوعي

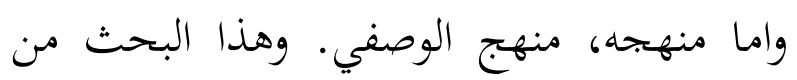
دراسة الحالة تستخدم الباحثة هذا المنهج والمدخل لتصف مشكلات ترقية كفاءة الطلبة في اللغة العربية في قسم تعليم اللغة العربية بالجامعة سلطان

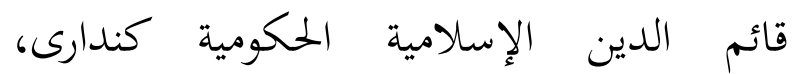
واحتاجت الباحثة إلى تحليل وتفسير عميق عنها لنيل المعلومات الواضحة والمعبرة عن العوامل الداعمة والعائقة في استراتيجية ترقية كفاءة الطلبة في اللغة العربية في قسم تعليم اللغة العربية ولتحليل مشكلات وحلولها في ترقية كفاءة الطلبة في اللغة العربية في قسم تعليم اللغة العربية بالجامعة سلطان قائم الدين الإسلامية الحكومية

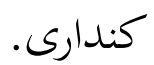

في هذا البحث، تكون الباحثة أداة من

ادوات البحث لبحثها. فكان لزاما عليها ان تراكز البحث، وتختار المجيبين كمصادر البيانات، وبتحمع البيانات وتحلل وتفسر وتستنتج حتى تجد نتائج

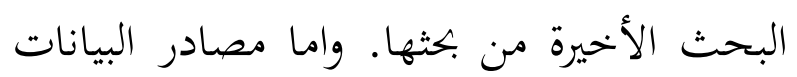
هي من رئيس قسم تعليم اللغة العربية ومحاضرى اللغة العربية وطلبة في قسم تعليم اللغة العربية. واستخدمت الباحثة الملاحظة والمقابلة والوثائق والإستبانة لاسلوب جمع البيانات في هذا البحث.

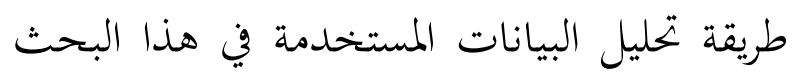
هو لتحليل الكيفي نموذج التحليل الكيفي للمايلز
وانطلاقا من ذالك فيُحتاج إلى استراتيجية

ترقية كفاءة الطلبة في اللغة العربية التي تعود على إنى سدّ احتياجات طلبة قسم تعليم اللغة العربية كحلول المشكلات الموجودة في قسن تعليم اللغة العربية بالجامعة الإسلامية الحكومية كندارى. اكمال مرافق التعلم، وترقية أداء عمل المحاضر في الفصل وزيادة دافعية الطلبة لتعمّق وتعلم اللغة العربية. إعطاء الاهتمام الخاص إلى العوامل الداعمة والعائقة في ترقية كفاءة الطلبة في اللغة العربية هي مهم لأن هذه العوامل هي العناصر الرئيسية في تخطيط استراتيجية ترقية كفاءة الطلبة في اللغة العربية. ومن هذه القضايا شعرت الباحثة بتحليله على اختيار الموضوع : "مشكلات ترقية كفاءة الطلبة في اللغة العربية" دراسة الحالة في قسم تعليم اللغة العربية بالجامعة الإسلامية الحكومية كندارى. واما مشكلات في هذا البحث هي : (1) كيف العوامل الداعمة والعائقة في استراتيجية ترقية كفاءة الطلبة في اللغة العربية في قسم تعليم اللغة العربية بالجامعة الإسلامية الحكومية كندارى ؟ (2) ماهي مشكلات وحلولها في ترقية كفاءة الطلبة في اللغة العربية في قسم تعليم اللغة العربية بالجامعة الإسلامية الحكومية

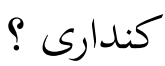


كاملا من اختيار المدخل والطريقة

ووسائل التعلم والمواد المناسبة بالطلبة

وزيادة على ذالك وجب على انتهاز

خصة الدروس والوظيفة المنزيلية

ودعامة التعلم لكي يستطيع الطلبة

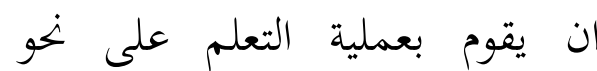

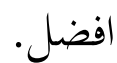

المحاضر هو المريّي والعلماء مع

المهمة الرئيسية لتحويل وتطوير ونشر

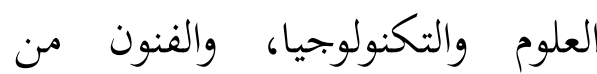

خلال التعليم والبحوث و وخدمة البحتمع (القانون رقم 2005/14)، وكما الأكاديميين ان المحاضر لديه الوظيقة لتحويل العلوم والتكنولوجيا مايتعمّق فيه للطلبة في التعلم ويجعل مناخ التعلم حتى يتمكن الطلبة بنشاط على تطوير وترقية كفاءقم (القانون رقم 2012/12).

وهذا يصف على حسن من

هداء عمل المحاضر مايشتمل من اختيار الإستراتيجية المناسبة وطريقة ووسائل التعلم، لكن ولو يكون نتائج الطلبة في تعلم اللغة العربية جيدا لكن في الحقيقة هناك المشكلات التي وجب على المحاضر ان يهتمه كاستعمال وسائل التعلم التكنولوجيا
وهوبرمان التفاعلية. عرض مايلز وهوبرمان أن تحليل البيانات الكيفيبوصفية حينما إعطاء معنى للبيانات التي تم جمعها وتحليلها وتفسير البيانات. يقوم بتحليل البيانات، أولا، جمع حتى ينتهي

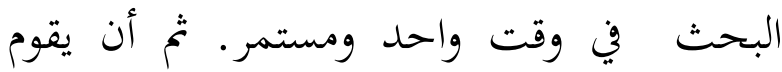
التأويل والتفسير البيانات بالإشارة إلى المرجع النظرية المتعلقة والمتصلة بمشاكل البحث. تتكون تحليل البيانات ما يلي: 1 1) تخفيض البيانات، 2). عرض البيانات، 3). أخذ الإستنتاج. 3

نتائج البحث وتحليلها ومناقشها

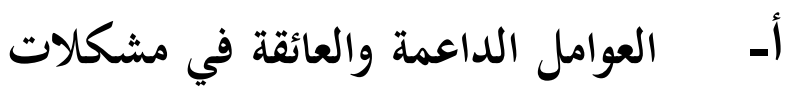
ترقية كفاءة الطلبة في اللغة العربية بالجامعة الإسلامية الحكومية كندارى. العوامل الداعمة والعائقة في مشكلات ترقية كفاءة الطلبة في اللغة العربية بالجامعة الإسلامية الحكومية كندارى هي : ترفهة

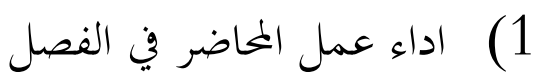

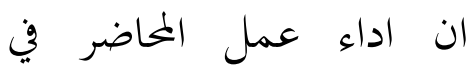
الفصل له دور مهم في ترقية كفاءة الطلبة في اللغة العربية، وليس الاّ في

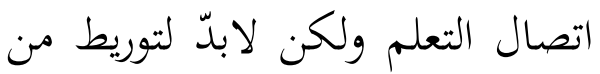
جميع احوال عملية تعلم الطلبة. لذلك وجب على المحاضر اعدّ اعدادا

${ }^{3}$ M. Junaidi Ghony dan Fauzan Almanshur, Metodologi Penelitian Kualitatif, hlm.306. 
أداء عمل المحاضر في الفصل

هو مهم، وليس فقط في بحال

الاتصالات في التعلم ولكن ينبغي أن

يشتمل من جميع جوانب أنشطة تعلم

الطلبة. لذلك لابد ليعدّ المحاضر

اعدادا جيدا وهو تعمّق على مواد

التعلم، وفهم خصائص الطلبة، فهم

أهداف التعلم وتحديد استراتيجية

التعلم المناسبة وإدارة الفصل ما ماسل يشتمل من ظروف ومناخ التعلم.

وان معدل نتائج الطلبة

لدروس اللغة العربية اها جيد جدا

لان تصل النتائج فوق 3 وهي درس

النحو 3.3 ودرس الصرف 3.3

ودرس المحادثة 3.6 ودرس المطالعة

3.5 وكذلك اها تصف عن جيدة

إستراتيجية التعلم المستخدمة بالمحاضر

في الفصل قد رقّى على كفاءة الطلبة

$$
\text { في اللغة العربية. }
$$

كقول ديتا ايو وينرسيه في

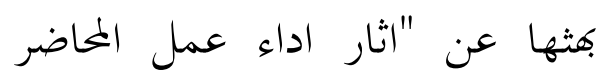

ومهارة التعليم على نشاط التعلم

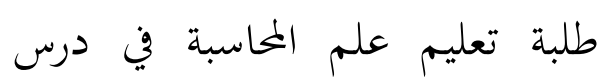

القنون الخاص والتجارة" ان اداء عمل

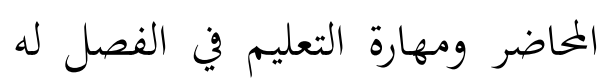

اثار إيهابي على نشاط تعلم الطلبة
التي لم يتم استخدامها في تعلم اللغة

العربية بقسم تعليم اللغة العربية في

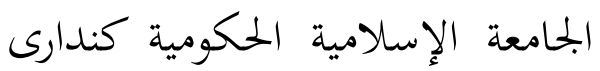

تستطيع ان تساعد المحاضر في زيادة

رغبة الطلبة لاهتمام شرح مواد التعلم

وكذلك استخدام الإستراتيجية

المتعددة والمتفرقة لكل لقاء الدرس الإسترديه

وليس استخدام متساوية الإستراتيجية

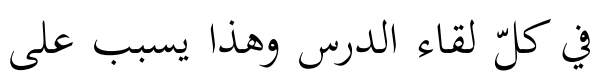

غير مساعد في مناخ التعلم الذي

يؤثر على دافعية الطلبة في التعلم.

وين مستوى التطبيق في

الفصل ان اداء عمل المحاضر

وخصائص الطلبة سيكون عاملا

رئيسيا في فعالية عملية التعلم، وقوة

وسلطة المحاضر فيه لا يمكن أن فيسيه

يستخدم كأساس لإملاء كيفية عملية

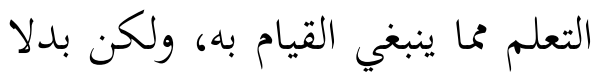

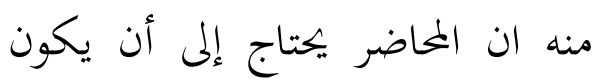

حريصا على فهم خصائص الطلبة

ومستندة نظام التعلم في الفصل نهو

الطلبة، لأنه في سياق النظام، ان

الطلبة هو من الذي ينبغي أن يتعلم

ويتم تعليمه، وكذلك ان الطلبة هو

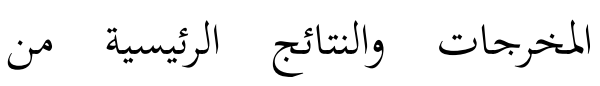

التعليم العالي التي تتم بها الجامعة. 
حول 21.13 والفرقة المراقبة عدم

استخدام معمل اللغة يجصل على

نتيجة 10.78، وهذا يسمى ان

تعلم باستخدام معمل اللغة يساعد

على ترقية كفاءة الطلبة في اللغة

$$
\text { العربية. }
$$

والمكتبة هي بحموعةٌ منظمةٌّة

من مصادر المعرفة تكون متاحةً

لمحتمع معرّف من من أجل البحث والاطلاع و والاستعارة. 4 وتنتاج المكتبة المدرسية الفرصة أمام الطلبة لاستخراج المعلومات بأنفسهم وهذا ما يساعدهم على إثراء مبدأ التعليم الذاتي. وتساعد على تنشئة الطلبة من تعمل على تنمية المهارات وتوجيهـا خلال أهدافها التعليمية ففي البحال التربوي والاستعانة بالقراءة والاطلاع لتنمية ميول الطلبة القرائية. والمكتبة تساعد على الطلبة في تعلم ذاتيا عن اللغة العربية، لكن ان لكنيه

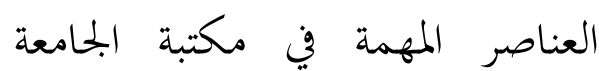
الإسلامية الحكومية كندارى هي كتب اللغة العربية لم يكن كاملا وهذا يؤثر على ترقية كفاءة الطلبة في اللغة

${ }^{4}$ https://ar.wikipedia.org/wiki/مكتبة/ diakses pada tanggal 2 November 2015
ويبين انه يعطى 18,6\% واما بقيه

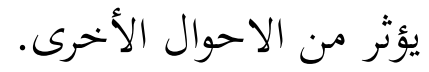
المرافق المساعدة في تعلم اللغة العربية

ان المرافق المساعدة في تعلم

اللغة العربية تساعد الطلبة في ترقية كفاءقم في اللغة العربية حين خصة التعلم وكذلك خارج خصة التعلم كاستخدام معمل اللغة لتعلم درس المحادثة او لتدريب استماع الطلبة من ناطق العربي. لكن معمل اللغة في تعلم اللغة العربية لم يكن مساعدا في ترقية كفاءة الطلبة في اللغة العربية بقسم تعليم اللغة العربية في الجحامعة

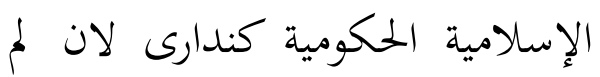
يجقق استخدامه كاحد من وسائل التعليمية المساعدة في ترقية كفاءة الطلبة في اللغة العربية. وان تعلم اللغة باستخدام معمل اللغة له تفريق بشكل بارز مع تعلم اللغة بدون استخدام معمل اللغة كقول يويون ايفا مولن في رسالتها عن "اثار في استخدام معمل اللغة على مهارة الإستماع طلبة الفصل السابع في المدرسة المتوسطة الحكومية ونوكرومو" لإسطية ان في الفرقة التجريبية باستخدام معمل اللغة يكصل على ترقية نتيجة 
وان دافعية طلبة قسم تعليم

اللغة العربية في تعلم اللغة العربية جيد

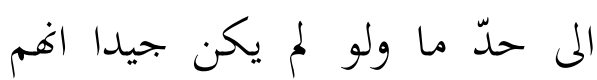

يتبعون على كلّ الأنشطة اداها بقسم

تعليم اللغة العربية وكذلك اهما دور

مهم في مساعدة ترقية كفاءقم في

اللغة العربية. وفي زيادة دافعية الطلبة

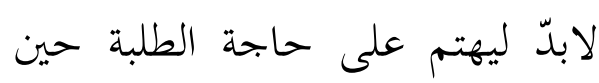

التعلم في الفصل ومايحتاجه خارج

الفصل. كداخل الفصل يمتاج الطلبة

على الإستراتيجية المتعددة التي

ستستخدم بالمحاصر لكي يبعد من الإنديه

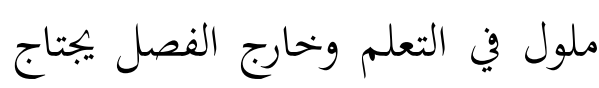
الطلبة على ميدان في تطبيق العلوم

التي تعلّمواها دخل الفصلى ميدان في لطبيق العلوم

وجود متلاحق ومتواصل التعلم يجعل

على عادة التعلم ذاتيا لان التعلم

الذاتي يجعل على حفظ العلوم طويلا

في ذهنه وهذا يؤثر دافعية الطلبة في

ترقية كفاءقم في اللغة العربية لان

$$
\text { تعلم اللغة يعود على العادة. }
$$

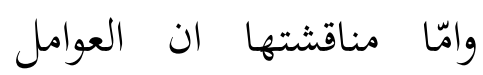

المساعدة في ترقية كفاءة الطلبة في

$$
\text { اللغة العربية هي: }
$$

1) اداء عمل المحاضر في الفصل انه

العوامل المساعدة مباشرة عند
العربية لان يؤدى الى قليل مصادر

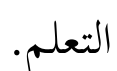

وان مؤسسة اللغة تكون

المرافق المساعدة في تعلم اللغة العربية

لان لها دور في ترقية كفاءة الطلبة في

اللغة العربية خارج الفصل وهي

باداءها تجعل المقرّرات الإضافية التي

تستطيع ان يستمر ما نالوهم في

الفصل وتطبيقها في المقرّرات الإضافية

وكذلك في بيئة اللغة واهما لم يحققها

بقسم تعليم اللغة العربية او بتعاون

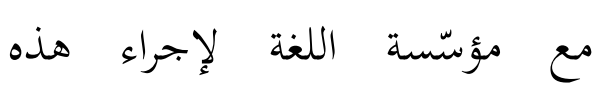
المقرّرات الإضافية والبيئة اللغوية.

دافعية الطلبة في تعلم اللغة العربية

يمكن على تفكير في أساليب

زيادة الدافعية المستخدمة في حجرة

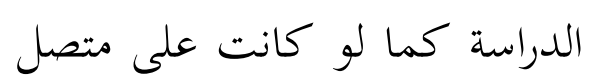

من الداخل والخارج، فأساليب زيادة

الدافعية الداخلية هي التي ترتبط

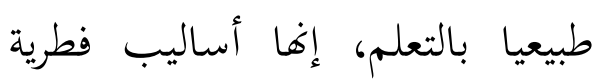

سواء في عملية التعلم ذاها أو في

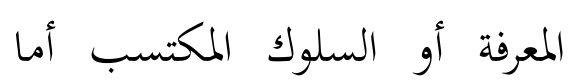

أساليب زيادة الدافعية الخارجية فهي المعركي

من الأنشطة تتعلق باللغة العربية او الوالية

$$
\text { المقرّرات الإضافية. }
$$


والعوامل السلبية في ترقية

كفاءة الطلبة في اللغة العربية هي :

1) اذا لم يكن تحقيق المرافق في تعلم

اللغة العربية فيصير على سلبية في

ترقية كفاءة الطلبة في اللغة العربية

لانّ تسبب على ناقص الميدان

لتطبيق و التعلم الذاتي عن اللغة

العربية عند الطلبة فتؤثر على

دافعية الطلبة في ترقية كفاءهم في

اللغة العربية كما شعر بطلبة قسم

تعليم اللغة العربية في الجامعة

$$
\text { الإسلامية الحكومية كندارى }
$$

2) ناقص دافعية الطلبة تسبب على الإسلى نداريه

ناقص ارادة الطلبة في تعمّق اللغة

العربية وكذلك تؤثر على اتساقهم

في مرابطة محاولتهم في ترقية كفاءة

الطلبة وهذا يسبب من قليل

ميدان في تطبيق اللغة العربية.

المشكلات وحلولها في ترقية كفاءة

الطلبة في اللغة العربية في قسم تعليم

اللغة العربية بالجامعة الإسلامية

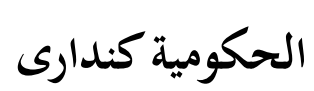

ومن بيانات البحث و وجدت

الباحثة مشكلات ترقية كفاءة الطلبة في

اللغة العربية في قسم تعليم اللغة العربية

بالجامعة الإسلامية الحكومية كندارى
الطلبة في ترقية كفاءقم في اللغة

العربية لان المحاضر هو الذي

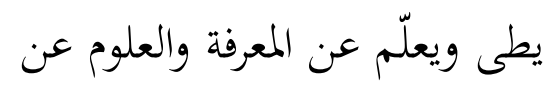

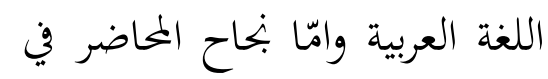

تعليم اللغة العربية يدلّ على بنحاح

إستراتيجية التعلم المستخدمة في

الفصل. وكانت إستراتيجية التعلم

المستخدمة بمحاضر قسم تعليم

اللغة العربية بالجامعة الإسلامية

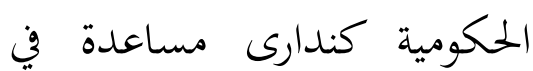

ترقية كفاءة الطلبة في اللغة العربية

في الفصل وهذا يدلّ على نتيجة

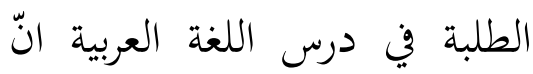

معدّلها جيدا وهو فوق 3.00

2) المرافق المساعدة في تعلم اللغة

العربية كمعمل اللغة والمكتبة

ومؤسّسة اللغة والبيئة اللغوية

وكلّها تستطيع ان تساعد في ترقية

كفاءة الطلبة في اللغة العربية لاها

جرت على عادة الطلبة في تعلّم

ذاتيا وتطبيق اللغة العربية خارج

الفصل، ومعاودة على تعلّم ذاتيا

وتطبيق اللغة العربية تحعل على

ثابت العلوم وكذلك ترقية كفاءة

الطلبة في اللغة العربية. 
وانطلاقا مما ذكر من بيات سابق

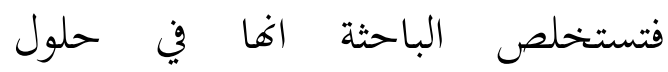

المشكلات ترقية كفاءة الطلبة في اللغة

العربية في قسم تعليم اللغة العربية بالجامعة

الإسلامية الحكومية كنداري لابدّ لينظرها

من جهة حاجات الطلبة، لكي يحقق

أهداف قسم تعليم اللغة العربية فعالية،

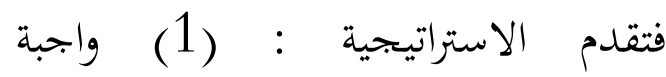

استعمال اللغة العربية على المحاضر والطلبة

في الفصل وخارج الفصل (2) اجرت الندوة عن تعليم اللغة العربية للمحاضر

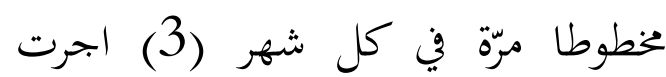
البيئة اللغوية (4) تعاون بمؤسّسة اللغة في سدّ الأنشطة والمقرّرات الإضافية في ترقية كفاءة الطلبة في اللغة العربية يوميا كصناعة بحلة الحائط، واسبوعيا كمقابلة اللغة العربية، وشهريا كسباق المحادلة بين الفترة (5) اكمال مرافق تعلم اللغة كمعمل اللغة وكتب اللغة العربية.

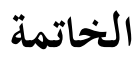

بعد ان قامت الباحثة بعرض البيانات

وتحليلها ومناقشتها فتصل على الملخص الاتية : 1- العوامل المساعدة والسلبية في إستراتيجية ترقية كفاءة الطلبة في اللغة العربية في قسم
وهي: من قليل الأنشطة المتعلقة باللغة العربية لطلبة قسم تعليم اللغة العربية التي تسبب على قليل ميدان لتطبيق اللغة العربية ولم تكن المرافق المساعدة في تعلم اللغة العربية تحقيقا ومستخدما لتساعد على ترقية كفاءة الطلبة في اللغة العربية كعدم البيئة اللغوية لانّ اكث الطلبة يشعر بسخيف لاستعمال اللغة العربية

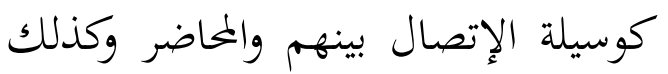

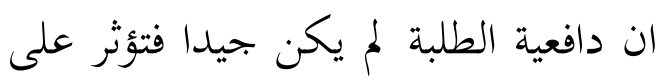
عدم الإتساق في تعلم اللغة العربية وتعمقها واغا تسبب من قليل الأنشطة المتعلقة باللغة العربية وان قسم تعليم اللغة العربية لم يكن اتساقا في اجراء الأنشطة او البرنامج المتعلقة باللغة العربية كبرنامج مقابلة اللغة العربية ووجب على حلى حلول المشكلات وهي كحماية النظام الجيد لتعلم اللغة (العربية والإبحليزية) لمدّة فترتين عند الطلبة الجحديدة هي واحد من حلول المشكلات من نقص الميدان لتعلم اللغة هن العربية او حماية فرقة تعلم الطلبة ويدعمه لكي يكون فرقة تعلم الطلبة ان تبقى دائما لان تعلم بين الطلبة فصار على لئي زيادة شجاعة ان يسأل شيء الذي لانيه لايفهم وكذلك يجعل حالا مريها للتعلم. 
العلوم وكذلك ترقية كفاءة الطلبة في

$$
\text { اللغة العربية. }
$$

والعوامل السلبية في ترقية كفاءة

$$
\text { الطلبة في اللغة العربية هي : }
$$

1) اذا لم يكن تحقيق المرافق في تعلم

اللغة العربية فيصير على سلبية في

ترقية كفاءة الطلبة في اللغة العربية

لانّ تسبب على ناقص الميدان

لتطبيق والتعلم الذاتي عن اللغة العربية

عند الطلبة فتؤثر على دافعية الطلبة

في ترقية كفاءقم في اللغة العربية كما

شعر بطلبة قسم تعليم اللغة العربية

في الجامعة الإسلامية الحكومية

$$
\text { كندارى. }
$$

2) ناقص دافعية الطلبة تسبب على

ناقص ارادة الطلبة في تعمّق اللغة

العربية وكذلك تؤثر على اتساقهم في

مرابطة محاولتهم في ترقية كفاءة الطلبة

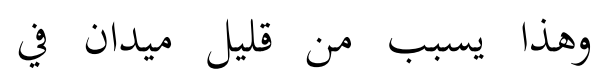

$$
\text { تطبيق اللغة العربية. }
$$

ومن العوامل الداعمة والعائقة في

ترقية كفاءة الطلبة في اللغة العربية وجدت

الباحثة انّ إستراتيجية ترقية كفاءة الطلبة

في اللغة العربية في قسم تعليم اللغة العربية

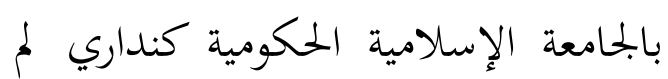

تعليم اللغة العربية بالجامعة الإسلامية

$$
\text { الحكومية كنداري }
$$

1) اداء عمل المحاضر في الفصل انه

العوامل المساعدة مباشرة عند الطلبة

في ترقية كفاءقم في اللغة العربية لان

المحاضر هو الذي يطى ويعلّم عن

المعرفة والعلوم عن اللغة العربية وامّا

بحاح المحاضر في تعليم اللغة العربية

يدلّ على بناح إستراتيجية التعلم

المستخدمة في معلى الفصل. و وكانت

إستراتيجية التعلم المستخدمة بمحاضر

قسم تعليم اللغة العربية بالجامعة

الإسلامية الحكومية كندارى مساعدة

في ترقية كفاءة الطلبة في اللغة العربية

في الفصل وهذا يدل على نتل نتيجة

الطلبة في درس اللغة العربية انّ معدّها

$$
\text { جيدا وهو فوق } 3.00
$$

2) المرافق المساعدة في تعلم اللغة العربية

كمعمل اللغة والمكتبة ومؤسّسة اللغة

والبيئة اللغوية وكلّها تستطيع ان تساعد

في ترقية كفاءة الطلبة في اللغة العربية

لاها جرت على عادة الطلبة في تعلّم

ذاتيا وتطبيق اللغة العربية خارج

الفصل، ومعاودة على تعلّم ذاتيا

وتطبيق اللغة العربية تجعل على ثابت 
الفصل وخارج الفصل (2) اجرت الندوة

عن تعليم اللغة العربية للمحاضر مخطوطا مرّة في كل شهر (3) اجرت البيئة اللغوية

(4) تعاون بمؤسّسة اللغة في سدّ الأنشطة

والمقرّرات الإضافية في ترقية كفاءة الطلبة

في اللغة العربية يوميا كصناعة مجلة

الحائط،

واسبوعيا كمقابلة اللغة العربية،

وشهريا كسباق البحادلة بين الفترة (5)

اكمال مرافق تعلم اللغة كمعمل اللغة

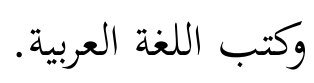

\section{المراجع العربية - ماية}

أوريل بحر الدين. مهارات التدريس نحو إعداد

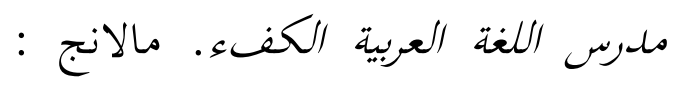

مطبعة جامعة مولانا مالك إبراهيم

$$
\text { الإسلامية، } 2011
$$

ثائر أمد غباري وأصحابه. البحث النوعي في

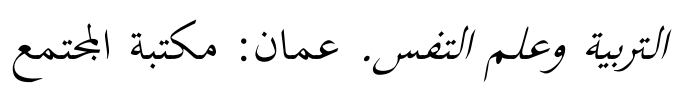

$$
\text { العربي، } 2011
$$

رجاء وحيد دويدري. البحث العلمي. دمشق:

$$
\text { دار الفكر، رحاء وحيل دويلري. }
$$

$$
\text { المراجع الأجنبية }
$$

Ghony, M. Junaidi dan Fauzan Almanshur,

Metodologi Penelitian Kualitatif.

Yogyakarta: Ar-Ruzz Media, 2012
2- المشكلات وحلولها في ترقية كفاءة الطلبة في

اللغة العربية في قسم تعليم اللغة العربية

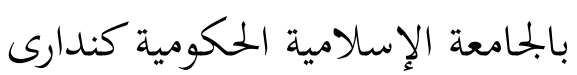

ان قسم تعليم اللغة العربية في ترقية

كفاءة الطلبة في اللغة العربية وجد

المشكلات التي وجبت على حلولها، وهي

1) قليل الأنشطة او البرامج المتعلقة باللغة

$$
\text { العربية }
$$

2) اتساق في اجراء الأنشطة او البرامج

المتعلقة باللغة العربية

3) ان المرافق المساعدة في تعلم اللغة العربية

لم تكن تحقيقها

4) ان دافعية الطلبة لم تكن جيدة فتئثر على

اتساقهم في تعلم اللغة العربية وهذا تسبب

من قليل الميدان لتطبيق اللغة العربية

وتعلمها ذاتيا.

وان حلول المشكلات الموجودة في

ترقية كفاءة الطلبة في اللغة العربية في قسم

تعليم اللغة العربية بالجامعة الإسلامية

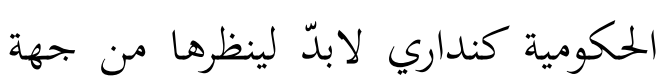

حاجات الطلبة، لكي يمقق أهداف قسم لهم لهن تعليم اللغة العربية فعالية، فتقدم

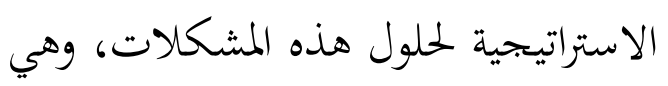

:

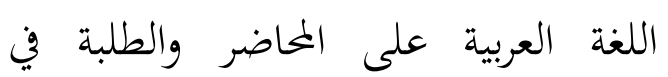


Suharsaputra, Uhar. Manajemen Penddidikan Perguruan Tinggi. Bandung:PT Refika Aditama, 2015

Sukardi. Metodolgi Penelitian pendidikan. Jakarta:Bumi Aksara, 2009

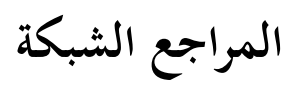

http://ar.wikipedia.org/wiki/مكتبة/ مكية
Qamar, Mujamil. Dimensi Manajemen Pendidikan Islam. Jakarta:PT Erlangga, 2015

Satori, Djamaan dan Aan Komariah. Metodologi Penelitian Kualitatif. Bandung: Alfabeta, 2011

Sugiyono. Metode Penelitian Pendidikan, Pendekatan Kuantitaif, Kualitatif, dan $R \& D$. Cet-20. Bandung:Alfabeta, 2014. 